\title{
What's up with the rubber hand illusion?
}

\author{
Anil Seth ${ }^{1,2,4}$, Warrick Roseboom ${ }^{1,2,3}$,Zoltán Dienes ${ }^{1,3}$, and Peter Lush ${ }^{1,3}$ \\ ${ }^{1}$ Sackler Centre for Consciousness Science, University of Sussex, Brighton, BN1 9QJ, UK \\ 2 School of Engineering and Informatics, University of Sussex, Brighton, BN1 9QJ, UK \\ ${ }^{3}$ School of Psychology, University of Sussex, BN1 9QH, UK \\ ${ }^{4}$ Canadian Institute for Advanced Research (CIFAR) Program on Brain, Mind, and \\ Consciousness, Toronto, Ontario, Canada
}

\begin{abstract}
The rubber hand illusion (RHI) is a cornerstone of the scientific literature on embodiment. We have recently published a series of studies investigating the RHI, in particular its relationship to hypnotic (imaginative) suggestibility, and the validity of commonly used control conditions. These studies have generated substantial discussion regarding our claims, how they should be interpreted, and what all this means for past and future experimental studies of embodiment experiences [see, for example, (Makin, 2020)]. To clarify these issues, here we first summarise our main points (there is of course much more in the papers) and then offer responses to some frequently asked questions.
\end{abstract}

\subsection{Introduction}

In the RHI, synchronous stroking of a person's real hand, which is hidden from view, and a (suitably positioned) rubber hand can elicit reports that the rubber hand, in some sense, 'feels like' the person's real hand (Botvinick \& Cohen, 1998). When the stroking is asynchronous, such reports are typically weaker. On this basis, standard accounts of this phenomenon appeal to multisensory integration [see (Braun et al., 2018; Ehrsson, Holmes, \& Passingham, 2005) reviews]. These accounts have also been motivated by, or extended to account for, a variety of related observations in RHI experiments, such as changes in perceived hand position (proprioceptive drift), alterations in skin conductance and temperature, as well as neuroimaging evidence [see, for example (Blanke, 2012)].

The Sussex studies address both the subjective report aspect of the RHI paradigm (such as the tendency of participants to agree with the statement 'I felt as if the rubber hand were my hand' (Botvinick \& Cohen, 1998), and more 'objective' indices such as 'proprioceptive drift' (the rated spatial displacement of the real hand from its actual physical location). Importantly, RHI studies that make claims about subjective experiences of ownership typically do so solely in virtue of their connection to subjective reports. Thus, it is important to be clear about their basis. 
Our studies were motivated by the idea that there might be an inadequately controlled factor confounding ownership reports in the RHI: demand characteristics [(Orne, 1962), see Figure 1]. Broadly, this is the long-standing worry in psychology that characteristics of experimental design may lead people to 'know the correct answer' that the experimenter is 'looking for' - and that this knowledge, or expectation, whether implicit or explicit, may explain their responses.

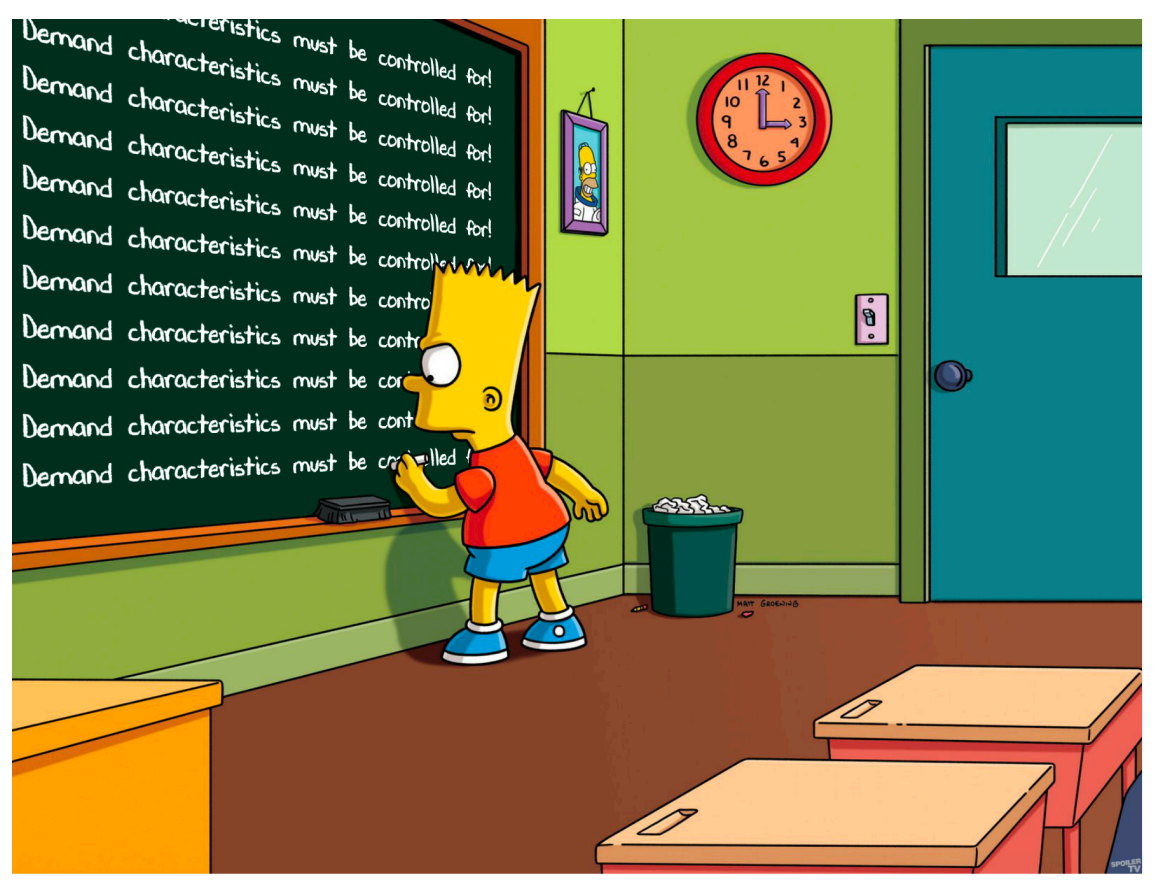

Figure 1. The importance of controlling for demand characteristics.

Importantly, demand characteristics may exert their effects not only through participants merely making responses according to what they think the experimenter wants ('behavioural compliance'), but also by actually shaping or generating subjective experiences to fit experimental demands (Kirsch \& Council, 1989; Olson, SuissaRocheleau, Lifshitz, Raz, \& Veissiere, 2020). Importantly, demand characteristics may influence different people in different ways. In particular, people who are more capable of producing experiences consistent with task demands in a hypnotic context, may also be able to respond to demand characteristics in general, outside of that context, by changing their actual experience and its underlying neurophysiological basis (Dienes, Palfi, \& Lush, 2020).

We hypothesised that if demand characteristics influence the RHI then people who score higher on a scale of hypnotic/imaginative suggestibility should experience the illusion more strongly. Our first study tested this hypothesis in a large sample of 353 participants (Lush et al., 2020). In an exploratory analysis, we found that suggestibility (or more generally what we call trait 'phenomenological control', PC for short) 
correlated substantially with reported experiences of ownership as measured by questionnaire responses; trait PC also correlated with proprioceptive drift.

However, we also found that the differences in reported ownership between synchronous (sync) and asynchronous (async) stroking remained robust across levels of trait PC. We had attempted to change expectancies with direct instructions (i.e., short 'cover stories' suggesting that async stroking would lead to stronger illusory embodiment). However, we were unable to shift participants' expectancies this way. This meant that a pre-registered element of this first study - which had proposed the hypothesis that suggestion effects could 'invert' the RHI - could not be tested. We reasoned that this manipulation failed because expectations provided by the nature of the paradigm itself concerning sync and async stroking may be too strong to be overcome by short cover stories provided by the experimenter.

To test this reasoning, in a second study we directly probed peoples' expectations about experiences of owning a rubber hand in the two conditions (sync and async) (Lush, 2020). To be clear, in this study we did not actually implement the RHI, but instead asked people what they would expect to happen, after carefully describing the setup and procedure. Strikingly, we found that people expected the same pattern of responses, across sync and async conditions, that is typically observed in RHI studies. This was true for both subjective responses and for proprioceptive drift. The fact that people have strong a priori expectations for what to experience in these conditions plausibly explains why short cover stories did not substantially alter peoples' experience of the RHI. More importantly, this experiment made clear that contrasting sync vs async stroking is not sufficient to control for demand characteristics in the RHI.

In a third study, we explored how unmeasured inter-individual variability in suggestibility might affect conclusions drawn from RHI studies that have used standard, relatively small ( $\sim 20$ participants or fewer) sample sizes (Roseboom \& Lush, 2020) (https://psyarxiv.com/uhdzs). We analysed a range of simulated RHI experiments, varying the proportion of the sample that was high in PC. Here, we found that samples with a majority of participants high in PC almost guarantee finding evidence for the RHI, while experiments with samples comprised only of participants low in PC will find evidence for the RHI only around half the time (in fact only around $4 \%$ of the time when considering ratings explicitly about "ownership"). This simulation study substantiates the possibility that experiments using the RHI may be studying trait PC by proxy; or equally, the role of top-down influences of the sort involved in PC in generating and/or shaping experiences of embodiment.

Altogether, our studies reveal that individual differences in suggestibility (trait PC) confound subjective measurements of ownership in the RHI. Contrasting sync vs async stroking (or existing control statements and illusion statements) is not sufficient to control for demand characteristics, and - importantly - the inadequacy of this contrast 
to control for demand characteristics applies independently of our demonstrated influence of imaginative suggestibility. It is an issue for RHI studies very generally, and likely also an issue of other related 'embodiment illusions' such as the 'full body illusion' (Ehrsson, 2007; Lenggenhager, Tadi, Metzinger, \& Blanke, 2007) and the 'enfacement illusion' (Sforza, Bufalari, Haggard, \& Aglioti, 2010) - though these possibilities remain to be directly tested.

We highlight three further points arising from our studies. First, we do not claim to have demonstrated that the RHI is 'explained away' by imaginative suggestibility (trait PC). It remains an open question as to whether factors besides implicit suggestion, in the form of demand characteristics, are driving subjective reports and/or proprioceptive drift. Second, addressing this open question requires the development of adequate control conditions. For example, if we wish to test multisensory integration accounts, we need to compare conditions which vary in their reliance on multisensory integration mechanisms, but which are matched for participants' expectations about what to experience. In addition, because imaginative suggestion effects vary in difficulty it will also be necessary to control for suggestion difficulty. See (Lush, 2020).

Finally, our finding that individual differences in PC correlate with subjective reports is, we believe, of substantial independent interest. It is likely that all perceptual experiences - whether of the world, or the body - rest on a combination of bottom-up (sensory) and top-down (expectation-based) factors. The idea that perception depends on an interaction between such factors is central to frameworks like predictive processing (Clark, 2013; Seth, 2013). Building on the idea of PC as intentional cognitive and motor actions that occur without awareness of these intentions (Dienes, Lush, et al., 2020; Dienes, Palfi, et al., 2020), we propose that imaginative suggestions can be construed as a distinctive species of top-down expectation within a predictive processing framework [see (Martin \& Pacherie, 2019) for a similar idea, regarding experiences of agency]. Unpacking the details of how PC generates perceptual experience is an exciting avenue for future research, in the RHI and beyond (e.g. synaesthesia; Lush et al. 2020).

\subsection{Frequently Asked Questions}

We now address some frequently asked questions. Some responses cover the same ground as above in more encapsulated form, while others cover additional common queries and concerns.

\section{Q1. "Are you saying the rubber hand illusion doesn't exist?"}

No, we are not saying that. When people report illusory experiences of ownership, it is entirely plausible that they have the experiences they say they have. We are arguing that current studies cannot exclude that these experiences are due to implicit 
imaginative suggestion effects - to phenomenological control (PC). We have shown that such suggestion effects play a role. Whether suggestion is all that's going on remains an open question.

\section{Q2. "Why do you claim existing literature is uninterpretable?"}

It is uninterpretable with respect to causes in changes in experiences of ownership. Evidence for changes in experience in the RHI comes from subjective reports. Embodiment researchers acknowledge the dangers uncontrolled demand characteristics present for interpretation of subjective report and have used control methods (typically asynchronous stroking conditions and control statements) for over two decades to claim that effects cannot be attributed to suggestion effects or demand characteristics. These methods are invalid - as we show, they do not control for demand characteristics, but in fact follow from them. Therefore, reported changes in experience may be attributable to demand characteristics and suggestion effects.

\section{Q3. "Why do you insist on throwing the baby out with the bathwater?"}

We are not throwing the baby out with the bathwater. Our goal is precisely the opposite. We wish to understand more about the mechanisms and processing underlying empirical phenomenological, behavioural, (neuro)physiological observations in the RHI, as well as in other related phenomena (e.g. mirror touch synaesthesia and vicarious pain, Lush et al., 2020). Zooming out, we wish to understand the role top-down expectations play in the construction of perceptual experiences of both the body and the world.

\section{Q4. "We already have a control condition (asynchronous stroking) that rules out an} influence of suggestion effects"

Unfortunately not. Synchronous and asynchronous stroking conditions differ in demand characteristics, such that participants implicitly know how to respond in each case. If participants expect to respond in one condition but not in another, any difference between these conditions may be attributable to suggestion effects. We tested this by asking people what they would expect to experience in each condition, having fully described the procedure. We found that peoples' expectations about the synchrony manipulation matched what people actually report when this manipulation is done in practice (Lush, 2020).

Q5. 'Don't most RHI studies report the difference between synchronous and asynchronous stroking conditions? But your study shows that this difference is not affected by phenomenological control - so there's no problem!"

As above, people have different expectancies for synchronous versus asynchronous stroking, so this difference too could be due to demand characteristics. Also, the 
majority of RHI studies in fact report and interpret subjective responses in the synchronous stroking condition only, as evidence for the illusion, even in cases where data have been collected from both synchronous and asynchronous stroking conditions [see (Roseboom \& Lush, 2020)].

Q6. "The correlation reported between RHI measures and suggestibility measures is too low for you to say that RHI measures are completely confounded"

We do not claim that suggestibility accounts for everything. We claim that there is not yet evidence that it doesn't. Interpretation of variance explained between two scales requires consideration of reliability of both scales. Neither is measured perfectly, so high variance-explained cannot be expected even if there is perfect correlation in the underlying constructs. Our simulation studies further examine the extent of the confound, from the perspective of gaining evidence for a RHI (Roseboom \& Lush, 2020).

Even with noise in measurement, a useful indication of the strength of the relation is in terms of raw effect sizes. For example, for the crucial synchronous condition, each unit increase in hypnotic response was associated with a 0.3 unit increase in subjective response on the illusion statements (on a -3 , completely disagree, to +3 , completely agree, scale); the mean score was +1.3 . Likewise, the degree of proprioceptive drift increased by $0.6 \mathrm{~cm}$ for each unit increase in hypnotic response, while the mean amount of drift across the entire sample was only $1.1 \mathrm{~cm}$. See (Lush et al., 2020).

\section{Q7. "What about the implicit measures like proprioceptive drift?"}

Although proprioceptive drift is described as 'implicit' or 'objective' it is more accurate to describe it as 'indirect'. We found the trait PC correlates with reported proprioceptive drift (Lush et al., 2020). Whether people have different expectancies about proprioceptive drift in synchronous versus asynchronous conditions (as we they do about explicit subjective reports) is something we are currently testing - our initial data suggests that indeed they do.

\section{Q8. "What about physiological changes (e.g. skin conductance)?"}

The idea that physiological measures are immune from suggestibility is incorrect. There is in fact considerable evidence that physiological changes, such as the skin conductance responses, can be altered through hypnotic suggestion. In one highly cited paper (1681 citations, Google Scholar, 13/01/2021), Levenson and colleagues report voluntary changes in skin conductance relating to changes in facial expression (Levenson, Ekman, \& Friesen, 1990). Furthermore, imaginative suggestion has been repeatedly demonstrated over more than half a century to affect electrodermal activity (Barber \& Coules, 1959; Kekecs, Szekely, \& Varga, 2016). It is also notable that imaginative suggestion can drive changes in fMRI measures and histamine reactivity, as we describe in (Lush, 2020). Altogether, any apparently 'implicit' or 'objective' measure cannot be 
assumed to be unaffected by suggestibility; it needs to be shown to be unaffected through the development of fit-for-purpose control conditions.

Q9. "Are we saying that phenomenological control causally drives subjective reports in the RHI?"

This is our working hypothesis. Presently, the data are consistent. Until there are contradictory data, this hypothesis stands. We welcome all attempts to challenge it. We found a correlation between trait PC and strength of ownership responses in the RHI. What we can say with confidence is that trait PC predicts such responses (as in the title of (Lush et al., 2020)), and the reverse is of course true too. We of course recognise that correlation is not by itself sufficient to establish causation (see Figure 2).
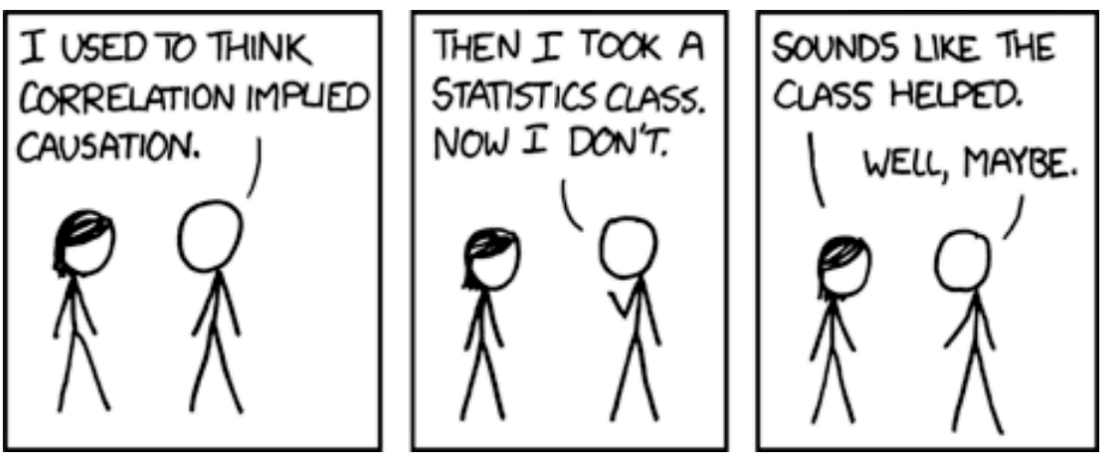

Figure 2. Correlation does not establish causation. From https://xkcd.com/552/.

Q10. "There is so much convergent evidence. How can suggestion effects explain animal results like the 'rubber tail illusion' in mice?"

There is a rich body of interesting work on body perception and representation in nonhuman animals [see, for example (Catania \& Remple, 2002; Fang et al., 2019; Wada, Takano, Ora, Ide, \& Kansaku, 2016)]. Our studies in no way invalidate behaviourist approaches that seek to map body perception to neural function through observed behaviour. However, instances where these findings are related to subjective experiences of ownership through their connection with RHI-like paradigms are problematic, because - as shown by our studies - the changes in reported experience in RHI-like paradigms may be due to demand characteristics. In short, if claims about human subjective body ownership are confounded by some other variable, then all subsequent inferences grounded on these claims - whether in humans or other animals - are brought into question. A related question is whether implicit demand characteristics could play also play a role in animal experiments. Addressing this question requires a deeper understanding of how demand characteristics exert their effects in humans. These issues are discussed further in (Roseboom \& Lush, 2020).

Q11. "But of course body perception is based on multisensory perception and therefore the RHI results from multisensory integration - this is trivial" 
Sure, but this doesn't mean claims that you 'own' a rubber hand stand up. Body perception and 'ownership experience' aren't the same thing. Again, our results do not demonstrate that multisensory integration is not involved in the RHI, merely that existing studies do not provide sufficient evidence that ownership reports are not explicable in terms of suggestion effects. We are open to the possibility that the RHI can provide such evidence. Future research into how confounding demand characteristics can be effectively controlled will be required to settle this question.

It is also important to recognise that illusory embodiment experiences in the RHI - even for highly suggestible people - are a far cry from the altered experiences in clinical conditions such as somatoparaphrenia and xenomelia. Participants in the RHI are not amazed when it is revealed that the rubber hand is not in fact their hand. People with somatoparaphrenia are often convinced that their limb does in fact belong to someone else (Vallar \& Ronchi, 2008), while people with xenomelia sometimes go as far as selfamputation in an attempt to resolve aberrant experiences of embodiment (Hilti et al., 2013). It could well be that disturbances in multisensory integration underwrite altered embodiment experiences in clinical conditions, while demand characteristics underwrite the subjectively weaker altered embodiment experiences in the RHI.

Q12. "What about evidence of body ownership from clinical cases like somatoparaphrenia? You can't explain that with suggestion effects"

Our data have nothing to say about such clinical evidence except where it interacts with the RHI paradigm. More generally, as mentioned above, illusory embodiment experiences in the RHI - even for highly suggestible people - are typically subjectively weaker than altered experiences of embodiment in clinical conditions such as somatoparaphrenia, or xenomelia. Returning to an earlier (baby and bathwater) point, we are nowhere claiming that there is no such thing an experience of embodiment, or that such experiences cannot be altered.

Q13. "Psychophysical methods show that participants are sensitive to small discrepancies in space or time with RHI. They can't possibly be influenced by demand characteristics"

This interpretation of the psychophysical methods used is incorrect. Single interval yes/no or 2 response alternative forced choice tasks (sometimes referred to as 2 alternative forced choice) are not immune to the influence of demand characteristics. The measures derived from these tasks such as point of subjective equality (or subjective simultaneity in a simultaneity judgement) conflate decisional and perceptual influences. Differences between conditions, such as between synchronous and asynchronous stroking conditions (or other contrasts of 'illusion' and 'control' conditions) alone provide no evidence for whether the change is due to decision or perceptual biases. See Chapter 2 of (Kingdom \& Prins, 2009) for a more general discussion of limitations on interpreting psychophysical tasks. 
Q14. 'Could suggestibility also play a role in other 'embodiment illusions', such as the full body illusion, the enfacement illusion, and the like?"

We have not directly tested this. But given that these 'illusions' are based on methods very similar to the RHI the answer is likely yes.

\section{Q15. "You are just being negative. Why are you trying to destroy this field? Contribute something to the discussion."}

We are not trying to destroy the field, we are trying to enhance it. We pointed out where a commonly used paradigm was being misinterpreted, provided extensive evidence in support of this claim, and suggested ways to improve experimental paradigms to overcome the problems we identified.

We have also offered a substantial alternative theory that applies over a broad range of effects: That people can use a capacity for PC to dramatically alter how they experience the world (Dienes, Lush, et al., 2020; Dienes, Palfi, et al., 2020). The theory is general, testable, it may impact many areas in psychology and everyday life, and though it based in a strong and empirically rigorous traditions it seems to many people surprising. Little attention has been paid to the other two effects reported in our correlations paper (vicarious pain and mirror-touch synaesthesia) (Lush et al., 2020). Our claim is not specific to the RHI, the RHI just happens to be one of the first three effects in which predictions of our theory has been tested.

Our studies open many new interesting questions, such as: (i) to what extent are empirical findings in the RHI driven by PC? (ii) to what extent are they driven by multisensory integration? (iii) how do these two factors interact? (iv) are there analogs of PC in non-human animals? (v) how, mechanistically, does PC exert its effects? (vi) how do RHI experiences differ from clinical cases of altered embodiment experiences, and to what extent can these differences be understood in terms of different roles for PC? (vii) what kinds of experiences - embodied or otherwise - are immune to PC? We are sure these questions merely scratch the surface of what is on offer.

\section{Q16. "It's such a huge and influential field. How can it be wrong?"}

It is not a question of being 'wrong'. It is a question of being open to enriched explanations, even if this makes experiments harder to do and casts doubt on previous interpretations. Our work has not broken the existing RHI controls. We have merely explained why they are not fit for purpose, and showed this to be the case. They would be unfit for purpose whether we had pointed it out or not. Zooming out, there have been many occasions in psychology where overly simplistic explanations have been found wanting. Ego depletion was a huge field. Mirror neurons were a huge topic. Sometimes, as a community, we fail to see the whole story. In fact we never see the whole story, and that is the point. The important thing is how we respond - by acknowledging and addressing the identified problems, or by persisting with problematic paradigms. 


\subsection{Summary}

The rubber hand illusion literature exemplifies both a problem and an opportunity for psychological research that relies on subjective report for establishing 'ground truth'. The problem is that experimental designs must control for demand characteristics across experimental conditions, otherwise it cannot be excluded that differences between conditions are due to demand characteristics either driving behavioural compliance and/or subjective experience, through phenomenological control. The opportunity is that phenomenological control offers a unique experimental window onto how 'top-down' influences shape and/or generate behaviour and subjective experience. It is likely that many phenomena, including but by no means limited to experiences of embodiment, causally involve top-down expectations manifesting through demand characteristics and phenomenological control. Mapping this terrain and unravelling the underlying mechanisms will shed new light on the neurocognitive basis of conscious experiences in general.

\section{Acknowledgements}

The authors are grateful to the Dr Mortimer and Theresa Sackler Foundation, which supports the Sackler Centre for Consciousness Science. AKS and PL are also grateful for support from the Canadian Institute for Advanced Research. An earlier version of this paper previously appeared on AKS's blog:

https://neurobanter.com/2020/11/09/whats-up-with-the-rubber-hand-illusion/

\section{$\underline{\text { References }}$}

Barber, T. X., \& Coules, J. (1959). Electrical skin conductance and galvanic skin response during "Hypnosis". International journal of Clinical and Experimental Hypnosis, 7, 7992.

Blanke, O. (2012). Multisensory brain mechanisms of bodily self-consciousness. Nature reviews. Neuroscience, 13(8), 556-571. doi:10.1038/nrn3292

Botvinick, M., \& Cohen, J. (1998). Rubber hands 'feel' touch that eyes see. Nature, 391(6669), 756. doi:10.1038/35784

Braun, N., Debener, S., Spychala, N., Bongartz, E., Soros, P., Muller, H. H. O., \& Philipsen, A. (2018). The Senses of Agency and Ownership: A Review. Front Psychol, 9, 535. doi:10.3389/fpsyg.2018.00535

Catania, K. C., \& Remple, M. S. (2002). Somatosensory cortex dominated by the representation of teeth in the naked mole-rat brain. Proc Natl Acad Sci U S A, 99(8), 5692-5697. doi:10.1073/pnas.072097999

Clark, A. (2013). Whatever next? Predictive brains, situated agents, and the future of cognitive science. Behav Brain Sci, 36(3), 181-204. doi:10.1017/S0140525X12000477

Dienes, Z., Lush, P., Palfi, B., Roseboom, W., Scott, R. B., Parris, B. A., . . Lovelll, M. (2020). Phenomenological control as cold control: Hypnosis and beyond. APA Psychology of Consciousness: Theory, Research, and Practice. 
Dienes, Z., Palfi, B., \& Lush, P. (2020). Controlling phenomenology by being unaware of intentions. In J. Weisberg (Ed.), Qualitative consciousness: Themes from the philosophy of David Rosenthal. Cambridge: Cambridge University Press.

Ehrsson, H. H. (2007). The experimental induction of out-of-body experiences. Science, 317(5841), 1048.

Ehrsson, H. H., Holmes, N. P., \& Passingham, R. E. (2005). Touching a rubber hand: feeling of body ownership is associated with activity in multisensory brain areas. J Neurosci, 25(45), 10564-10573. doi:10.1523/JNEUROSCI.0800-05.2005

Fang, W., Li, J., Qi, G., Li, S., Sigman, M., \& Wang, L. (2019). Statistical inference of body representation in the macaque brain. Proc Natl Acad Sci U S A, 116(40), 2015120157. doi:10.1073/pnas.1902334116

Hilti, L. M., Hanggi, J., Vitacco, D. A., Kraemer, B., Palla, A., Luechinger, R., . . Brugger, P. (2013). The desire for healthy limb amputation: structural brain correlates and clinical features of xenomelia. Brain, 136(Pt 1), 318-329. doi:10.1093/brain/aws316

Kekecs, Z., Szekely, A., \& Varga, K. (2016). Alterations in electrodermal activity and cardiac parasympathetic tone during hypnosis. Psychophysiology, 53(2), 268-277. doi:10.1111/psyp.12570

Kingdom, F. A. A., \& Prins, N. (2009). Psychophysics: A practical introduction: Elsevier.

Kirsch, I., \& Council, J. R. (1989). Response expectancy as a determinant of hypnotic behavior. In N. P. Spanos \& J. F. Chaves (Eds.), Hypnosis: The cognitive behavioral perspective (pp. 360-379). Buffallo, NY: Prometheus press.

Lenggenhager, B., Tadi, T., Metzinger, T., \& Blanke, O. (2007). Video ergo sum: manipulating bodily self-consciousness. Science, 317(5841), 1096-1099.

Levenson, R. W., Ekman, P., \& Friesen, W. V. (1990). Voluntary facial action generates emotion-specific autonomic nervous system activity. Psychophysiology, 27(4), 363384. doi:10.1111/j.1469-8986.1990.tb02330.x

Lush, P. (2020). Demand characteristics confound the rubber hand illusion. Collabra Psychology, 6, 22.

Lush, P., Botan, V., Scott, R. B., Seth, A. K., Ward, J., \& Dienes, Z. (2020). Trait phenomenological control predicts experience of mirror synaesthesia and the rubber hand illusion. Nat Commun, 11(1), 4853. doi:10.1038/s41467-020-18591-6

Makin, S. (2020). Hypnosis experts cast doubt on famous psychological experiments. Scientific American.

Martin, J. R., \& Pacherie, E. (2019). Alterations of agency in hypnosis: A new predictive coding model. Psychol Rev, 126(1), 133-152. doi:10.1037/rev0000134

Olson, J. A., Suissa-Rocheleau, L., Lifshitz, M., Raz, A., \& Veissiere, S. P. L. (2020). Tripping on nothing: placebo psychedelics and contextual factors. Psychopharmacology, 237(5), 1371-1382. doi:10.1007/s00213-020-05464-5

Orne, M. T. (1962). On the social psychology of the psychological experiment: with particular reference to demand characteristics and their implications. American Psychologist, 17, 776-783.

Roseboom, W., \& Lush, P. (2020). Serious problems with interpreting rubber hand illusion experiments.

Seth, A. K. (2013). Interoceptive inference, emotion, and the embodied self. Trends Cogn Sci, 17(11), 565-573. doi:10.1016/j.tics.2013.09.007 
Sforza, A., Bufalari, I., Haggard, P., \& Aglioti, S. M. (2010). My face in yours: Visuo-tactile facial stimulation influences sense of identity. Social neuroscience, 5(2), 148-162. doi:10.1080/17470910903205503

Vallar, G., \& Ronchi, R. (2008). Somatoparaphrenia: a body delusion. A review of the neuropsychological literature. Exp Brain Res.

Wada, M., Takano, K., Ora, H., Ide, M., \& Kansaku, K. (2016). The Rubber Tail Illusion as Evidence of Body Ownership in Mice. J Neurosci, 36(43), 11133-11137. doi:10.1523/JNEUROSCI.3006-15.2016 